\title{
Massive Ethylene Glycol Ingestion Treated with Fomepizole Alone-A Viable Therapeutic Option
}

\author{
Jennie A. Buchanan • Mohammed Alhelail • \\ Edward W. Cetaruk • Tammi H. Schaeffer • \\ Robert B. Palmer • Ken Kulig • Jeffrey Brent
}

Published online: 27 April 2010

(C) American College of Medical Toxicology 2010

\begin{abstract}
Fomepizole is used to treat and prevent toxicity from ethylene glycol poisoning. Treatment with fomepizole without hemodialysis in massive ethylene glycol ingestion has been rarely reported in the literature; however, published literature and practice guidelines recommend considering dialysis for ethylene glycol levels $>50 \mathrm{mg} / \mathrm{dL}$. We report a case of massive ethylene glycol ingestion resulting in the highest serum ethylene glycol concentration in a patient without ethanol co-ingestion who was treated with fomepizole and was not hemodialyzed. A 48-year-old male presented to the emergency department after reportedly ingesting $>1$ liter of antifreeze in an attempt at selfharm. He denied concomitant ethanol consumption. His initial presenting serum ethylene glycol level was $700 \mathrm{mg} /$ $\mathrm{dL}$, with normal renal function, and a metabolic acidosis
\end{abstract}

This report was presented as an abstract at the North American Congress of Clinical Toxicology, Toronto, ON, Canada, 2008, and has been published as an abstract in Clinical Toxicology.

J. A. Buchanan $(\bowtie) \cdot$ M. Alhelail • E. W. Cetaruk

T. H. Schaeffer $\cdot$ R. B. Palmer

Rocky Mountain Poison and Drug Center,

777 Bannock St. MC 0180,

Denver, CO 80204, USA

e-mail: jennie.buchanan3@dhha.org

J. A. Buchanan - M. Alhelail E. W. Cetaruk - T. H. Schaeffer •

R. B. Palmer $\cdot$ J. Brent

University of Colorado Denver School of Medicine,

Aurora, CO, USA

E. W. Cetaruk · T. H. Schaeffer • R. B. Palmer $\cdot$ K. Kulig • J. Brent

Toxicology Associates,

Denver, CO, USA

M. Alhelail

King Abdulaziz Medical City,

Riyadh, Saudi Arabia with a high anion gap. One hour after presentation, he was started on intravenous fomepizole. Treatment with fomepizole continued until the patient's plasma ethylene glycol concentration was $16 \mathrm{mg} / \mathrm{dL}$. His metabolic acidosis quickly resolved, he had no adverse reactions to the treatment, and his renal function remained normal. Ultimately, he was discharged to a psychiatric unit without sequelae. Published literature and practice guidelines suggests considering hemodialysis initiation in patients with an ethylene glycol level $>50 \mathrm{mg} / \mathrm{dL}$. This recommendation is anecdotally, rather than evidence, based. With the potential risks inherent in hemodialysis, our case provides evidence that treatment with fomepizole without hemodialysis appears to be a viable alternative option in patients with even extremely high plasma ethylene glycol concentrations as long as their renal function is intact.

Keywords Ethylene glycol · Fomepizole · Hemodialysis · Overdose

\section{Introduction}

Ethylene glycol is a common low volatility constituent in antifreezes, deicing, and brake fluids. In 2006, there were 5,343 ethylene glycol exposures reported to poison centers in the USA, resulting in 17 deaths and 152 major adverse outcomes [1]. The actual number of cases is probably far higher.

Although not highly toxic itself, ethylene glycol is metabolized by alcohol dehydrogenase (ADH) to metabolites, which can result in metabolic acidosis, renal injury, cerebral edema, multiorgan failure, and death.

The mainstay of treatment of ethylene glycol poisoning is inhibition of $\mathrm{ADH}$, supplemented by hemodialysis in 
Table 1 Laboratory values

\begin{tabular}{lllll}
\hline Serum values & Day1 & Day2 & Day3 & Day4 \\
\hline Ethylene glycol, mg/dL & 700 & 445 & 93 & 16 \\
Sodium, mEq/L & 135 & 143 & 137 & 135 \\
Anion gap, mEq/L & 20 & 16 & 9 & 12 \\
Bicarbonate, mEq/L & 18 & 22 & 25 & 25 \\
Blood urea nitrogen, mg/dL & 13 & 8 & 7 & 7 \\
Creatinine, mg/dL & 0.9 & 0.8 & 0.8 & \\
ABG pH at 9.5 h & 7.42 & & & \\
Osmolality & 531 & & & \\
Osmolar gap at 5 h, mOsm/L & 222.2 & & \\
Urine drug screen & Negative & & & \\
Acetaminophen and salicylate & Negative & & & \\
\hline
\end{tabular}

selected patients [2]. Traditionally, ADH inhibition was accomplished by the administration of the competitive substrate ethanol. However, ethanol treatment is fraught with difficulties and potential complications [3-8].

Fomepizole is an alcohol dehydrogenase inhibitor approved for the treatment of ethylene glycol poisonings in 2000. The pharmacokinetics of fomepizole is very predictable [9-11]. Because it is free of the potential adverse effects associated with ethanol [5, 9, 12-14], fomepizole therapy may obviate the need for hemodialysis in selected cases.

Current practice guidelines recommend considering hemodialysis in patients with renal failure, metabolic acidosis, electrolyte disturbances, hemodynamic instability, or plasma ethylene glycol concentrations $\geq 50 \mathrm{mg} / \mathrm{dL}$ [15]. However, several case reports [16-20] and a case series [13] have indicated that it is possible to forgo hemodialysis in patients with substantially higher ethylene glycol concentrations if they are treated with fomepizole.

We report a patient with a serum ethylene glycol concentration of $700 \mathrm{mg} / \mathrm{dL}$ effectively and safely treated with fomepizole alone. This represents the highest reported ethylene glycol level in a patient who did not co-ingest ethanol and was treated solely with fomepizole.

\section{Case Report}

A 48-year-old male with no significant past medical history presented to the emergency department after reportedly ingesting $>1$ liter of automotive product containing antifreeze in a self-harm attempt. Upon presentation, he was conscious, dysarthric, and reported a single episode of emesis. He denied ethanol co-ingestion. His initial vital signs were heart rate 70 beats per minute, blood pressure $168 / 90 \mathrm{mmHg}$, respiratory rate 27 per minute, temperature $37^{\circ} \mathrm{C}$, and he had an oxygen saturation of $94 \%$ on 21 administered by nasal cannula. The exact time of exposure was unknown, but it reportedly occurred as a single ingestion on the evening of emergency department arrival.

His presenting serum laboratory values were anion gap $20 \mathrm{mEq} / \mathrm{L}$, sodium $135 \mathrm{mEq} / \mathrm{L}$, bicarbonate $18 \mathrm{mEq} / \mathrm{L}$, creatinine $0.9 \mathrm{mg} / \mathrm{dL}$, and ethylene glycol $700 \mathrm{mg} / \mathrm{dL}$. Other values are shown in Table 1. One hour after presentation, a loading dose of $15 \mathrm{mg} / \mathrm{kg}$ of intravenous fomepizole was administered followed by $10 \mathrm{mg} / \mathrm{kg}$ every $12 \mathrm{~h}$ for a total of five doses. Fomepizole treatment continued until the patient's serum ethylene glycol concentration was $16 \mathrm{mg} / \mathrm{dL}, 72 \mathrm{~h}$ after the peak measurement. There were no treatment-related adverse events. The patient was discharged to the psychiatric service on day 4 with normal renal function (Table 1) and without sequelae of his poisoning.

The elimination profile of ethylene glycol was consistent with first order kinetics and an apparent half-life of $21 \mathrm{~h}$ (Fig. 1).

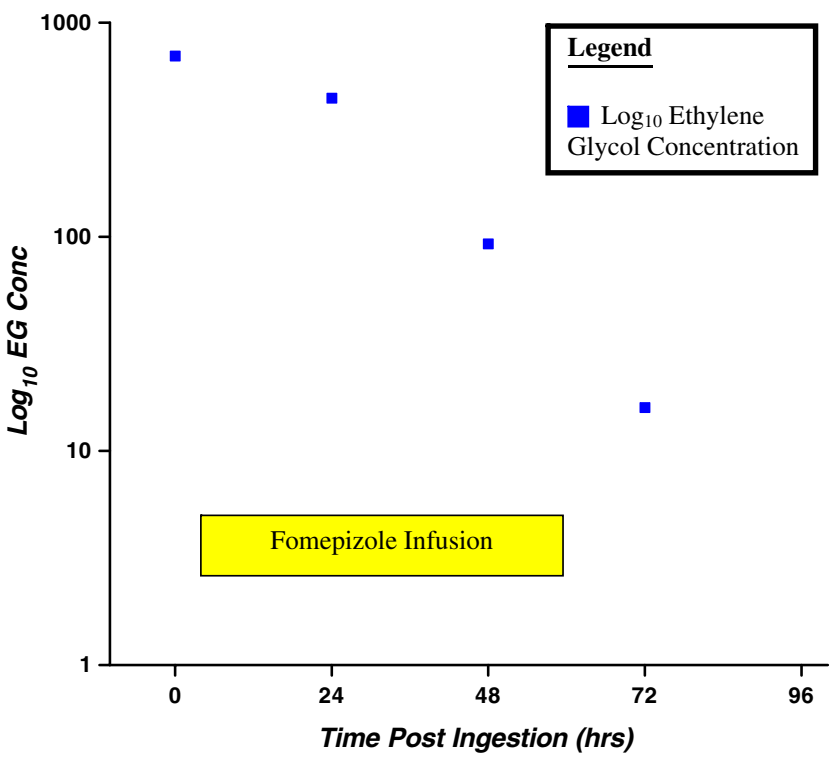

Fig. 1 Time course of serum ethylene glycol elimination. $\log _{10}$ ethylene glycol concentration over time 


\section{Discussion}

We report a case of massive ethylene glycol ingestion with an extremely high serum concentration in a patient presenting with normal renal function and mild metabolic acidosis. Because the patient had normal renal function and a mild metabolic acidosis at presentation, he was not dialyzed. The patient was successfully treated with fomepizole alone without hemodialysis, despite his very high serum ethylene glycol concentration. He did not develop signs of renal injury and had no adverse effects from fomepizole therapy.

Velez et al. reported a patient with an ethylene glycol level of $706 \mathrm{mg} / \mathrm{dL}$, normal renal function, and blood ethanol concentration of $84 \mathrm{mg} / \mathrm{dL}$ who was treated with fomepizole alone without adverse effect [16]; however, in contrast to our case, that patient co-ingested ethanol. In the META study, a prospective clinical trial of fomepizole use in ethylene glycol poisoned patients, those with normal renal function at the time of initiation of fomepizole treatment did not develop renal injury; however, hemodialysis was utilized in patients with plasma concentrations over $50 \mathrm{mg} / \mathrm{dL}$ [9]. Borron et al. reported a retrospective case series of 11 ethylene glycol poisoned patients treated with fomepizole [13]. Eight of these patients were not hemodialyzed. In that series, all patients with normal renal function at the time fomepizole therapy was started did not develop any subsequent signs of renal injury. These findings are consistent with the results of our case and those of the META trial [9].

Fomepizole inhibits $\mathrm{ADH}$ thus preventing oxidative metabolism of ethylene glycol [5]. The metabolic acidosis associated with ethylene glycol poisoning is due to the production of the metabolite glycolate, which is further metabolized to oxalate $[9,21,22]$. Injury to the kidneys and other organs caused by ethylene glycol poisoning is predominantly due to precipitation of calcium oxalate crystals [23]. Glycolate is dialyzable; however, it is unknown whether removal of glycolate by hemodialysis affects patient outcome in cases of ethylene glycol poisoning. Since our patient presented with a mild metabolic acidosis and did not sustain detectable renal injury, it is likely that some amount of glycolate may be well tolerated.

Fomepizole effectively inhibited ethylene glycol metabolism in our patient demonstrated by the resolution of his metabolic acidosis once fomepizole therapy was initiated. Despite inhibition of its metabolism, ethylene glycol can be effectively eliminated by renal clearance in the absence of kidney injury or failure [24]. This was demonstrated by the apparent first order elimination of ethylene glycol during fomepizole therapy (Fig. 1) and is consistent with the results of the META study [9]. Although previous studies demonstrated fomepizole therapy is an effective treatment for ethylene glycol poisoning [9, 13], they did not show efficacy in the absence of adjunctive hemodialysis in patients with very high levels. Our case adds to the mounting body of anecdotal evidence [7, 9, 16-20] that such patients may not require hemodialysis if their renal function is intact and they do not have a significant metabolic acidosis.

Although this patient did not have a blood ethanol determination on admission, he gave us a history of no ethanol ingestion, appeared to be a good historian, and did not have an odor of ethanol on his breath. A formal blood ethanol determination was done $24 \mathrm{~h}$ after admission and was negative $(<10 \mathrm{mg} / \mathrm{dL})$. Given that fomepizole would have blocked ethanol metabolism, it is likely that if he had substantial quantities in his blood at the time of admission, some would have still been detectable.

Hemodialysis was considered in our patient and that treatment might have shortened his hospital stay. However, hemodialysis has potential for complications, imparts additional costs, and affects resource allocation. Hemodialysis may not be available at all hospitals or may require long transport times to a center with this capability. Hemodialysis can also be technically challenging in small infants. Because of these factors, and based on the experience reviewed above, treatment of patients with fomepizole alone appears to be a viable option in those patients with very high serum ethylene glycol concentrations, without a severe metabolic acidosis, and normal renal function.

Funding support None.

\section{References}

1. Bronstein AC, Spyker DA, Cantilena LR Jr, Green J, Rumack BH, Heard SE (2007) Annual Report of the American Association of Poison Control Centers' National Poison Data System (NPDS). Clin Toxicol (Phila) 45:815-917

2. Jacobsen D (2005) Ethylene glycol and other glycols. In: Brent J, Wallace KL, Burkhart KK, Phillips SD, Donovan JW (eds) Critical care toxicology diagnosis and management of the critically poisoned patient. Elsevier Mosby, Philadelphia, pp 869-880

3. Hantson P, Wittebole X, Haufroid V (2002) Ethanol therapy for methanol poisoning: duration and problems. Euro J Emerg Med 9:278-279

4. Oker EE, Kanji SA, Aks S, Fishbein C, Crockett M (2001) Can health care workers effectively administer ethanol drips? Acad Emerg Med 8:443

5. Jacobsen D, McMartin KE (1997) Antidotes for methanol and ethylene glycol poisoning. Clin Toxicol 35:127-143

6. Palatnick W, Redman LW, Sitar DS, Tenenbein M (1995) Methanol half-life during ethanol administration: implications 
for management of methanol poisoning. Ann Emerg Med 26:202207

7. Boyer EW, Mejia M, Woolf A, Shannon M (2001) Severe ethylene glycol ingestion treated without hemodialysis. Peds 107:172-174

8. Salaspuro MP, Pikkarainen P, Lindros K (1977) Ethanol-induced hypoglycaemia in man: its suppression by the alcohol dehydrogenase inhibitor 4-methylprazole. Eur J Clin Investig 7:487-490

9. Brent J, McMartin K, Phillips S, Burkhart K, Donovan W, Wells $M$ et al (1999) Fomepizole for the treatment of ethylene glycol poisoning. Methylpyrazole for Toxic Alcohols Study Group. N Engl J Med 340:832-838

10. Wallemacq PE, Vanbinst R, Haufroid V, DiFazio V, Konig J, Detaille $T$ et al (2004) Plasma and tissue determination of 4methylpyrazole for pharmacokinetic analysis in acute adult and pediatric methanol/ethylene glycol poisoning. Ther Drug Monit 26:258-262

11. Jacobsen D, Barron SK, Sebastian CS, Blomstrand R, McMartin KE (1989) Non-linear kinetics of 4-methylpyrazole in healthy human subjects. Eur J Clin Pharmacol 37:599-604

12. Brent J, McMartin K, Phillips S, Aaron C, Kulig K (2001) Methylpyrazole for Toxic Alcohols Study Group. Fomepizole for the treatment of methanol poisoning. N Eng J Med 344:424-429

13. Borron SW, Mégarbane B, Baud FJ (1999) Fomepizole in treatment of uncomplicated ethylene glycol poisoning. Lancet 354:831

14. Megarbane B, Borron SW, Trout H, Hantson P, Jaeger A, Krencker E et al (2001) Treatment of acute methanol poisoning with fomepizole. Int Care Med 27:1370-1378

15. Barceloux DG, Krenzelok EP, Olson K, Watson W (1999) American Academy of Clinical Toxicology practice guidelines on the treatment of ethylene glycol poisoning. Ad Hoc Committee. J Toxicol Clin Toxicol 37:537-560

16. Velez LI, Sheperd G, Lee YC et al (2007) Ethylene glycol ingestion treated only with fomepizole. J Med Toxicol 3:125-128

17. Harry P, Jobard E, Briand M, Caubet A, Turcant A (1998) Ethylene glycol poisoning in a child treated with 4methylpyrazole. Peds 102:E31

18. Detaille T, Wallemacq P, Clement de Clety S, Vanbinst R, Dembour G, Hantson P (2004) Fomepizole alone for severe infant ethylene glycol poisoning. Pediatr Crit Care Med $5: 490-491$

19. Aakervik O, Svendsen J, Jacobsen D (2002) Severe ethylene glycol poisoning treated with fomepizole (4-methylpyrazole). Tidsskr Nor Laegeforen 122(25):2444-2446

20. Georg M, Al-Duaij N, Becker ML, Ewald MB (2008) Re: ethylene glycol ingestion treated only with fomepizole (Journal of Medical Toxicology: Volume 3, Number 3, September 2007; 125-8). J Med Toxicol 4(1):67

21. Jacobsen D, Ovrebo S, Ostborg J, Sejersted OM (1984) Glycolate causes the acidosis in ethylene glycol poisoning and is effectively removed by hemodialysis. Acta Med Scant 216:409-416

22. Clay KL, Murphy RC (1977) On the metabolic acidosis of ethylene glycol intoxication. Toxicol Appl Pharmacol 39:39-49

23. Corley RA, Wilson DM, Hard GC, Stebbins KE, Bartels MJ, Soelberg JJ et al (2008) Dosimetry considerations in the enhanced sensitivity of male Wistar rats to chronic ethylene glycol-induced nephrotoxicity. Toxicol Appl Pharmacol 228:165-178

24. Sivilotti ML, Burns MJ, McMartin KE, Brent J (2000) Toxicokinetics of ethylene glycol during fomepizole therapy: implications for management. For the Methylpyrazole for Toxic Alcohols Study Group. Ann Emerg Med 36:114-125 